\title{
Local heating with titanium nitride nanoparticles
}

Guler, Urcan; Ndukaife, Justus C.; Naik, Gururaj V.; Nnanna, A.G. Agwu; Kildishev, Alexander V.; Shalaev, Vladimir M.; Boltasseva, Alexandra

\section{Published in:}

Proceedings of 2013 Conference on Lasers and Electro-Optics (CLEO)

Link to article, DOI:

10.1364/CLEO_QELS.2013.QTU1A.2

Publication date:

2013

Document Version

Publisher's PDF, also known as Version of record

Link back to DTU Orbit

Citation (APA):

Guler, U., Ndukaife, J. C., Naik, G. V., Nnanna, A. G. A., Kildishev, A. V., Shalaev, V. M., \& Boltasseva, A. (2013). Local heating with titanium nitride nanoparticles. In Proceedings of 2013 Conference on Lasers and Electro-Optics (CLEO) [QTU1A.2] IEEE. https://doi.org/10.1364/CLEO_QELS.2013.QTu1A.2

\section{General rights}

Copyright and moral rights for the publications made accessible in the public portal are retained by the authors and/or other copyright owners and it is a condition of accessing publications that users recognise and abide by the legal requirements associated with these rights.

- Users may download and print one copy of any publication from the public portal for the purpose of private study or research.

- You may not further distribute the material or use it for any profit-making activity or commercial gain

- You may freely distribute the URL identifying the publication in the public portal 


\title{
Local heating with titanium nitride nanoparticles
}

\author{
Urcan Guler ${ }^{1}$, Justus C. Ndukaife, ${ }^{1,2}$, Gururaj V. Naik ${ }^{1}$, A. G. Agwu Nnanna², Alexander V. Kildishev ${ }^{1}$, \\ Vladimir M. Shalaev ${ }^{1}$, Alexandra Boltasseva ${ }^{1,3, *}$ \\ ${ }^{I}$ School of Electrical \& Computer Engineering and Birck Nanotechnology Center, Purdue University, West Lafayette, IN 47907, USA \\ ${ }^{2}$ Water Institute, Purdue University Calumet, Hammond IN, 46323, USA \\ ${ }^{3}$ DTU Fotonik, Department of Photonics Engineering, Technical University of Denmark, Lyngby, DK-2800, Denmark \\ *aeb@purdue.edu
}

\begin{abstract}
We investigate the feasibility of titanium nitride (TiN) nanoparticles as local heat sources in the near infrared region, focusing on biological window. Experiments and simulations provide promising results for $\mathrm{TiN}$, which is known to be bio-compatible.

OCIS codes: (250.5403) Plasmonics; (190.4870) Photothermal effects; (160.1435) Biomaterials
\end{abstract}

\section{Introduction}

Local heating with sub-wavelength particles has been intensely studied in the frame of various applications such as photothermal therapy and solar energy harvesting [1,2]. Not surprisingly, gold (Au) and silver (Ag) drew attention due to their plasmonic resonances in the visible region of electromagnetic spectrum [3]. However, for a variety of applications that require resonances in the near infrared (NIR) window, such as in vivo bio-imaging, serious efforts are needed in order to engineer particle geometry of noble metals and bring the resonance wavelengths into the region of interest [4].

Recently, we have shown that TiN nanoparticles provide high field enhancement in near field and strong absorption within the particle [5,6]. Local heating performance of a nanoparticle is directly related to absorption efficiency at the wavelength of interest. Hence, promising results obtained for absorption efficiency of TiN nanoparticles in NIR region lead us to investigate the local heating capabilities of TiN nanostructures. In this study, we examine the heat generation from TiN particles numerically and experimentally. Finite element method (FEM) based software (Comsol, Multiphysics) is employed so as to couple electromagnetic and heat transfer simulations. While illuminating with pulsed laser at various wavelengths, nanoparticle arrays fabricated by electron beam lithography (EBL) are examined with FLIR A320 infrared camera. When illuminated at resonance wavelength, particles absorb electromagnetic energy and act as nanometer-scale heat sources. Change in the temperature of the substrate and nanoparticle arrays are observed by the use of a thermal camera with spatial resolution of $25 \mu \mathrm{m}$.

Figure 1 (a) shows an illustration of nanodisk arrays used in this work. TiN and Au nanoparticles with identical geometric parameters are fabricated with EBL and examined both numerically and experimentally. Transmittance data simulated for these nanoparticles are given in Figure 1 (b), in which a strong but very narrow localized surface plasmon resonance (LSPR) dip can be observed for Au particles. Although the amplitude of plasmonic resonance is very strong for Au particles, spectral position and narrow width of resonance are drawbacks for biological applications. On the other hand, TiN LSPR resonance position is very well suited for bio-imaging. In addition, LSPR resonance in TiN nanoparticle is very broad, which is an advantage for bio-applications.
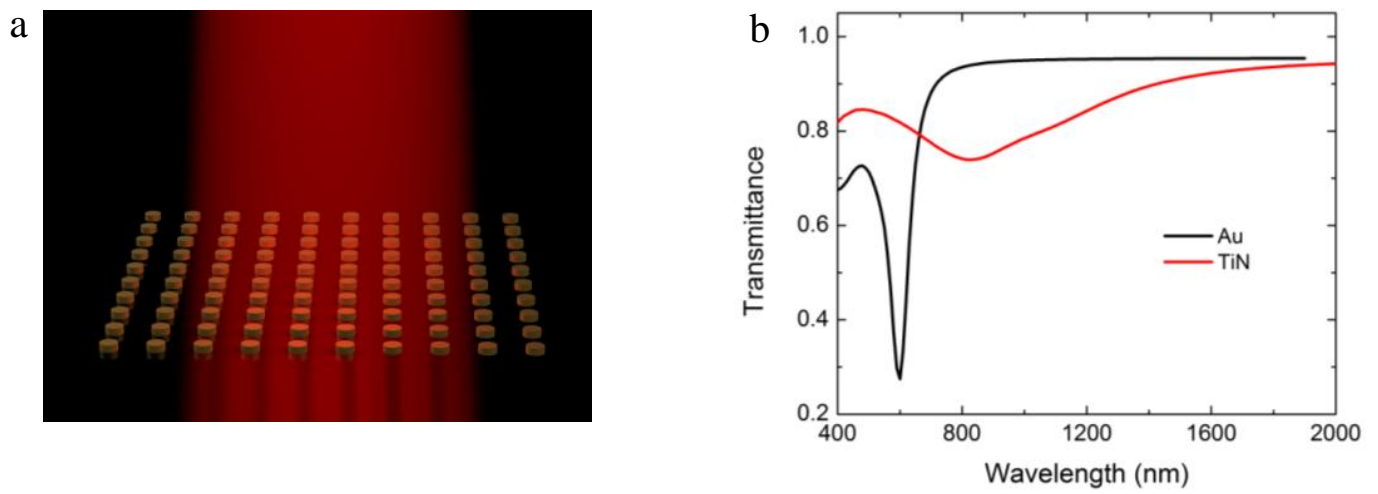

Fig. 1. (a) Illustration of nanodisk arrays. (b) Simulated transmittance of Au and TiN arrays located over glass substrate. 
Once the spectral positions of LSPR peaks are defined for both materials, nanoparticle arrays can be illuminated with laser pulses at several different wavelengths. Figure 2 (a) shows the thermal image of a TiN nanodisk array before illumination, at room temperature. Five different observation points are marked on the image. One of the observation points is in the middle of nanoparticle array while the others are distributed evenly away from the particles. Thermal image of the same area under laser illumination, after reaching steady state, is shown in Figure 2 (b). Position of the nanoparticle array can be easily determined from the contrast in thermal image after illumination.

Figure 2 (c) shows the temperature variation of each observation point in time. TiN particles are illuminated with laser light at $780 \mathrm{~nm}$, near LSPR wavelength, around five minutes and, then, left for cooling for another five minutes. Temperature increase up to $5{ }^{\circ} \mathrm{C}$ is measured at the nanoparticle array. Although the size of TiN particles and wavelength of illumination were not optimized, significant amount of temperature change is observed. Note the rapid drop in temperature when laser light is turned off at around $300 \mathrm{sec}$, which implies strong heat dissipation to environment.

a

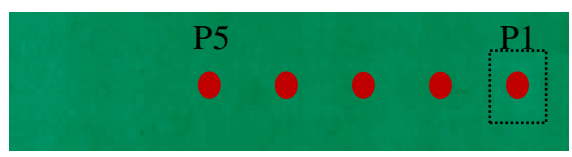

b

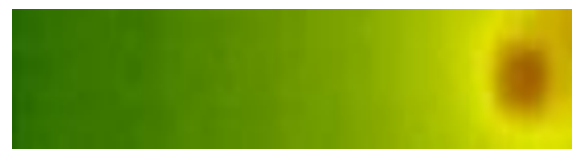

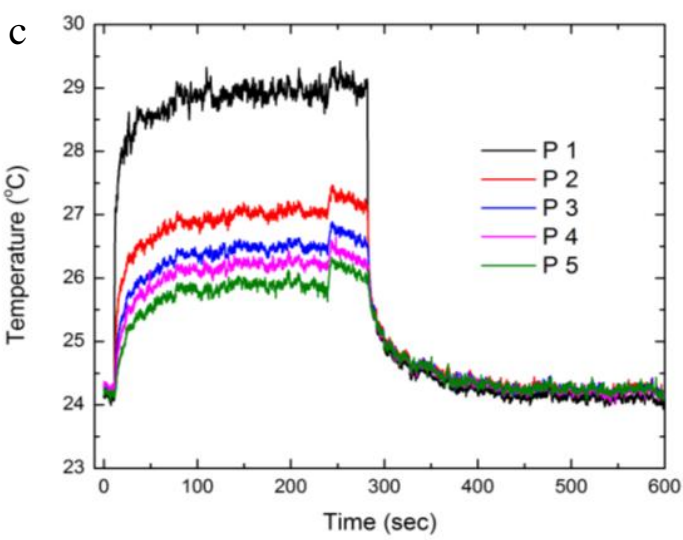

Fig. 2. (a) Thermal image of TiN nanoparticle array located over glass substrate at room temperature. Marks on the image show the observation points used in time dependent temperature plot, and dashed rectangle shows the position of nanoparticle array (b) Thermal image of the same area when the array is illuminated with pulsed laser light at $780 \mathrm{~nm}$ wavelength. (c) Time dependent temperature data for the five observation points located on the nanoparticle array and on the substrate.

In this contribution, both experimental and numerical results of local heat generation with TiN nanoparticles will be discussed. Obtained results will be compared with the Au nanoparticle arrays with identical geometric parameters. Results for a wide range of visible and NIR regions of the electromagnetic spectrum will be presented. It will be shown that TiN, being bio-compatible and CMOS compatible, is a promising material for local heating applications in the biological transparency window.

\section{References}

[1] E. Hutter and J. H. Fendler, "Exploitation of localized surface plasmon resonance," Adv. Mater. 16, 1685-1706 (2004).

[2] O. Neumann, A. S. Urban, J. Day, S. Lal, P. Nordlander, and N. J. Halas, "Solar vapor generation enabled by nanoparticles," ACS Nano 7, 4249 (2013)

[3] A. G. Brolo, "Plasmonics for future biosensors," Nat. Photon. 6, 709-713 (2012).

[4] S. Lal, S. Link and N. J. Halas, "Nano-optics from sensing to waveguiding," Nat. Photon. 1, 641-648 (2007).

[5] U. Guler, G. V. Naik, A. Boltasseva, V. M. Shalaev and A. V. Kildishev, "Performance analysis of nitride alternative plasmonic materials for localized surface plasmon applications," Appl. Phys. B 107, 285-291 (2012).

[6] U. Guler, G. V. Naik, A. Boltasseva, V. M. Shalaev and A. V. Kildishev, "Nitrides as alternative materials for localized surface plasmon applications," in Frontiers in Optics Conference, OSA Technical Digest (online) (Optical Society of America, 2012), paper FTh4A.2. 